VOL. $72(2005) \quad[39-44]$

\title{
A NONLINEAR MAP FOR MIDPOINT LOCALLY UNIFORMLY ROTUND RENORMING
}

\author{
S. Lajara and A.J. Pallars
}

We provide a criterion for midpoint locally uniformly rotund renormability of normed spaces involving the class of $\sigma$-slicely continuous maps, recently introduced by Moltó, Orihuela, Troyanski and Valdiva in 2003. As a consequence of this result, we obtain a theorem of $\mathrm{G}$. Alexandrov concerning the three space problem for midpoint locally uniformly rotund renormings of Banach spaces.

A normed space $(X,\|\cdot\|)$ (or its norm) is said to be midpoint locally uniformly rotund if for every $x \in X$ and every sequence $\left(x_{n}\right)_{n}$ in $X$ such that $\left\|x_{n}+x\right\| \rightarrow\|x\|$ and $\left\|x_{n}-x\right\| \rightarrow\|x\|$ we have $\left\|x_{n}\right\| \rightarrow 0$.

Recall also that $X$ is locally uniformly rotund if for every $x \in X$ and every sequence $\left(x_{n}\right)_{n} \subset X$ such that $\lim _{n}\left\|x_{n}\right\|=\|x\|$ and $\lim _{n}\left\|x_{n}+x\right\|=2\|x\|$ we have $\lim _{n}\left\|x_{n}-x\right\|=0$, and that $X$ is strictly convex or rotund ( $\mathrm{R}$ for short) if $x=y$ whenever $x$ and $y$ are elements of $X$ such that $\|x\|=\|y\|=\|(x+y) / 2\|$.

It is clear that locally uniformly rotund $\Rightarrow$ midpoint locally uniformly rotund and that midpoint locally uniformly rotund $\Rightarrow R$. In the paper [5], devoted to the renorming of spaces of continuous functions on trees, $R$. Haydon provides the first example (the only known to date) of midpoint locally uniformly rotund space with no equivalent locally uniformly rotund renorming. There, he also shows that for every tree $\Upsilon$, the existence of an equivalent strictly convex norm on $C(\Upsilon)$ implies midpoint locally uniformly rotund renormability on this space. This coincidence is not true in general: an example of strictly convexifiable space without midpoint locally uniformly rotund renorming is $\ell_{\infty}$ (see $[2,6])$.

Our aim in this paper is to provide a criterion for midpoint locally uniformly rotund renorming of spaces that have images in midpoint locally uniformly rotund spaces through special non linear maps. These are the $\sigma$-slicely continuous maps recently introduced in [11], where a non linear transfer technique for locally uniformly rotund renormability has been developed.

Received 7th February, 2005

Research supported by MCYT BFM 2002-01719 and CARM Sneca 00690-PI-04

Copyright Clearance Centre, Inc. Serial-fee code: 0004-9727/05 \$A2.00+0.00. 
Definition 1: Let $X$ and $Y$ be normed spaces, and let $A$ be a subset of $X$. A map $\Phi: A \longrightarrow Y$ is said to be $\sigma$-slicely continuous if for every $\varepsilon>0$ we may write $A=\bigcup_{n} A_{n, \varepsilon}$ in such a way that for every $x \in A_{n, \varepsilon}$ there exists an open half space $H \subset X$ such that $x \in H$ and $\operatorname{diam} \Phi\left(H \cap A_{n, \varepsilon}\right)<\varepsilon$.

Recall that an open half space of $X$ is a set of the form $\{x: f(x)>q\}$, with $q \in \mathbb{R}$ and $f \in X^{*} \backslash\{0\}$.

In [9] (see also [12]), there is a characterisation of locally uniformly rotund renormable spaces $X$ given in terms of countable decompositions of $X$ by sets which are union of slices with small diameter. From this characterisation it follows that, if $X$ or $Y$ is locally uniformly rotund renormable, then every linear bounded operator $Q: X \rightarrow Y$ is $\sigma$-slicely continuous, and the same holds for any composition $\Phi=B Q: X \rightarrow Z$ with $B: Y \rightarrow Z$ continuous (see [11, Proposition 1.14]).

We shall use $\sigma$-slicely continuous maps in order to obtain our main result as follows.

THEOREM 1. Let $X$ be a normed space. Suppose that there exist a $\sigma$-slicely continuous map $\Phi: X \rightarrow X$ and a midpoint locally uniformly rotund renormable subspace $Y$ of $X$ such that $x-\Phi x \in Y$, for all $x \in X$. Then, $X$ admits an equivalent midpoint locally uniformly rotund norm.

We apply this theorem to the three space problem for midpoint locally uniformly rotund renormability. The three space problem for a property of Banach spaces is the question whether a Banach space $X$ possesses this property provided that for some closed subspace $Y$ of $X$, both $Y$ and $X / Y$ have it. To admit an equivalent locally uniformly rotund norm is a three space property [4]. In the case of midpoint locally uniformly rotund (and rotund) renormings, the problem was negatively solved in [5]. It was however shown in [1] the following weak version of it, which is a consequence of Theorem 1.

THEOREM 2. Let $X$ be a Banach space, and suppose that there is a closed midpoint locally uniformly rotund renormable subspace $Y$ of $X$ such that the quotient $X / Y$ has an equivalent locally uniformly rotund norm. Then, $X$ admits an equivalent midpoint locally uniformly rotund norm.

Proof: We consider the quotient map $Q$, from $X$ onto $X / Y$. By the Bartle-Graves theorem (see for example, [3, Chapter VII.3]), there is a continuous selector $B: X / Y \rightarrow X$ such that $B Q x \in Q x$, for all $x \in X$. If we define $\Phi=B Q$, then $x-\Phi x \in Y$, for every $x \in X$. Since $X / Y$ is locally uniformly rotund renormable, $Q$ and $\Phi$ are $\sigma$-slicely continuous and Theorem 1 gives the midpoint locally uniformly rotund renormability on $X$.

Let us mention that a similar result to the previous was obtained in [7] for Kadec renormability (recall that a normed space is Kadec if the norm and the weak topologies coincide on its unit sphere).

An equivalent definition for midpoint locally uniformly rotund norms can be stated 
in terms of the notion of strongly extreme point of a subset of a normed space, introduced in $[8]$.

Definition 2: Let $A$ be a subset of a normed space $(X,\|\cdot\|)$, and $\varepsilon, \delta>0$. An element $x \in A$ is said to be an $(\varepsilon, \delta)$-strongly extreme point of $A$ if $\|u-v\|<\varepsilon$ whenever $u, v \in A$ and $\|x-(u+v) / 2\|<\delta$ The point $x$ is called an $\varepsilon$-strongly extreme point of $A$ it there exists $\delta>0$ such that $x$ is an $(\varepsilon, \delta)$-strongly extreme point of $A$.

A normed space is midpoint locally uniformly rotund if, and only if, every element of its unit sphere is an $\varepsilon$-strongly extreme point of the unit ball, for each $\varepsilon>0$.

In the proof of Theorem 1 we use the following covering type characterisation for the class of midpoint locally uniformly rotund renormable spaces given in [10, Theorem 1]: A normed space $X$ admits an equivalent midpoint locally uniformly rotund norm if, and only if, for every $\varepsilon>0$ we can write $X=\bigcup_{n} X_{n, \varepsilon}$ in such a way that each $x \in X_{n, \varepsilon}$ is an $\varepsilon$-strongly extreme point of $c o\left(X_{n, \varepsilon}\right)$.

Proof of Theorem 1: First step. The countable covering. Let us fix $\varepsilon>0$, and take the countable decomposition of the midpoint locally uniformly rotund renormable space $Y$ :

$$
Y=\bigcup_{n, m} Y^{n, m}
$$

with $n, m \in \mathbb{N}, 1 / m<\varepsilon / 2$, and such that every point $y \in Y^{n, m}$ is a $(\varepsilon, 1 / m)$-strongly extreme point of $\operatorname{co}\left(Y^{n, m}\right)$.

Let denote $\Psi(x)=x-\Phi(x) \in Y$, and lift the decomposition from $Y$ to $X$,

$$
X=\bigcup_{n, m} X^{n, m}
$$

where $X^{n, m}=\left\{x \in X: \Psi(x) \in Y^{n, m}\right\}$.

Now, we use the fact that $\Phi$ is $\sigma$-slicely continuous on each $X^{n, m}$ to get countable coverings

$$
X^{n, m}=\bigcup_{k, q} C_{k, q}^{n, m}
$$

with $k \in \mathbb{N}$ and $q \in \mathbb{Q}$, such that for each $x \in C_{k, q}^{n, m}$ there exist a positive rational number $r_{x}$ and an half space $H_{x}=\left\{y \in X: f_{x}(y)>q\right\}$, defined by $f_{x} \in X^{*}$ with $\left\|f_{x}\right\|=1$, that satisfy

$$
\operatorname{diam} \Phi\left(H_{x} \cap C_{k, q}^{n, m}\right)<\frac{1}{8 m}
$$

and $f_{x}(x)>q+\tau_{x}>q$. We can assume without loss of generality that $\Phi$ is bounded on $C_{k, q}^{n, m}$ baving

$$
\sup \left\{\|\Phi(x)\|: x \in C_{k, q}^{n, m}\right\}<M_{k, q}
$$

for some constant $M_{k, q}$. 
In order to get more control on the values of functionals $f_{x}$ we decompose a little more the pieces $C_{k, q}^{n, m}$. At this point we use some technical ideas taken from [10, Proposition 1] and [11, Lemma 4.21].

Let us consider, for each $x \in C_{k, q}^{n, m}$, a number $0<s_{x}<r_{x} /\left(16 m M_{k, q}\right)$, and define for each pair of positive rationals $r$ and $s$ the sets

$$
C_{k, q, r, s}^{n, m}=\left\{x \in C_{k, q}^{n, m}: s<s_{x} \text { and } q+r<\sup _{y \in C_{k, q}^{n, m}}\left\{f_{y}(x)\right\}<q+r+s\right\}
$$

Observe that for each $x \in C_{k, q, r, s}^{n, m}$ we have $r+s>r_{x}$.

Second step. A particular slice. Fix $x \in C_{k, q, r, s}^{n, m}$, take $y_{x} \in C_{k, q}^{n, m}$ such that

$$
q+r+s>f_{y_{x}}(x)>q+r>q \quad\left(x \in H_{y_{x}} \cap C_{k, q}^{n, m}\right)
$$

and consider the half space $H_{x}^{\prime}=\left\{z: f_{y_{x}}(z)>f_{y_{x}}(x)-r\right\}$.

For each $z \in C_{k, q, r, s}^{n, m} \cap H_{x}^{\prime}$ we have

$$
\begin{aligned}
& f_{y_{x}}(z)>f_{y_{x}}(x)-r>q \quad\left(z \in H_{y_{x}} \cap C_{k, q}^{n, m}\right) \\
& f_{y_{x}}(z)<q+r+s<f_{y_{x}}(x)+s \text { and } \\
& f_{y_{x}}(x)-f_{y_{x}}(z)>-s>-s_{x} .
\end{aligned}
$$

Inequalities (1), (2) and (3) ensure us that

$$
\|\Phi(x)-\Phi(z)\|<\frac{1}{8 m} .
$$

On the other hand, for $z \in C_{k, q, r, s}^{n, m} \backslash H_{x}^{\prime}$ we have

$$
f_{y_{x}}(x)-f_{y_{x}}(z) \geqslant r .
$$

Third step. A good estimation for convex combinations. Let us consider two convex combinations of vectors $u_{i} \in C_{k, q, r, s}^{n, m}(i \in I)$ and $v_{j} \in C_{k, q, r, s}^{n, m}(j \in J): u=\sum_{i \in I} \lambda_{i} u_{i}$ and $v=\sum_{j \in J} \mu_{j} v_{j}$, with $\lambda_{i}, \mu_{j}>0$ and $\sum_{i \in I} \lambda_{i}=\sum_{j \in J} \mu_{j}=1$.

Now, for any vector $x \in C_{k, q, r, s}^{n, m}$, we take the half space $H_{x}^{\prime}$ given in the above step, and define the subsets of indexes $I_{0}=\left\{i \in I: u_{i} \notin H_{x}^{\prime}\right\}$ and $J_{0}=\left\{j \in J: v_{j} \notin H_{x}^{\prime}\right\}$.

As in the proof of [10, Lemma 4], we take advantage of the inequalities (4) and (6) to obtain an upper bound for $\sum_{i \in I_{0}} \lambda_{i}+\sum_{j \in J_{0}} \mu_{j}$ :

$$
\begin{aligned}
\left\|x-\frac{u+v}{2}\right\| & \geqslant f_{y_{x}}\left(x-\frac{u+v}{2}\right) \\
& =\frac{1}{2}\left(\sum_{i} \lambda_{i}\left(f_{y_{x}}(x)-f_{y_{x}}\left(u_{i}\right)\right)+\sum_{j} \mu_{j}\left(f_{y_{x}}(x)-f_{y_{x}}\left(v_{j}\right)\right)\right)
\end{aligned}
$$




$$
\begin{aligned}
& >\frac{1}{2}\left(\sum_{i \in I_{0}} \lambda_{i}+\sum_{j \in J_{0}} \mu_{j}\right) r-\frac{1}{2}\left(\sum_{i \notin I_{0}} \lambda_{i}+\sum_{j \notin J_{0}} \mu_{j}\right) s \\
& =\frac{1}{2}\left(\sum_{i \in I_{0}} \lambda_{i}+\sum_{j \in J_{0}} \mu_{j}\right)(r+s)-s_{x} \\
& >\frac{1}{2}\left(\sum_{i \in I_{0}} \lambda_{i}+\sum_{j \in J_{0}} \mu_{j}\right) r_{x}-s_{x}
\end{aligned}
$$

From this inequality we deduce the following upper bound:

$$
\frac{1}{2}\left(\sum_{i \in J_{0}} \lambda_{i}+\sum_{j \in J_{0}} \mu_{j}\right)<\left(\left\|x-\frac{u+v}{2}\right\|+s_{x}\right) \frac{1}{r_{x}}
$$

Last step. Strongly extreme points. Now, we are going to prove that each point $x \in C_{k, q, r, s}^{n, m}$ is an $\varepsilon$-strongly extreme point of $c o\left(C_{k, q, r, s}^{n, m}\right)$. Thus, the cited covering charaterisation [10, Theorem 1] will finish the proof.

Let us fix $x \in C_{k, q, r, s}^{n, m}$ and take $0<\delta_{x}<1 /(16 m) \min \left\{1,\left(r_{x} / M_{k, q}\right)\right\}$. We shall prove that if $u$ and $v$ are in $\operatorname{co}\left(C_{k, q, r, s}^{n, m}\right)$ and $\|x-(u+v) / 2\|<\delta_{x}$, then $\|u-v\|<\varepsilon$.

We take $u=\sum_{i \in I} \lambda_{i} u_{i}$ and $v=\sum_{j \in J} \mu_{j} v_{j}$ with the same notation as in the previous step. Using that identity map is just the sum $\Phi+\Psi$, we get

$$
\begin{aligned}
\|u-v\| & =\| \sum_{i} \lambda_{i}\left(\Phi\left(u_{i}\right)+\Psi\left(u_{i}\right)\right)-\sum_{j} \mu_{j}\left(\Phi\left(v_{j}\right)+\Psi\left(v_{j}\right)\right) \\
& \leqslant\left\|\sum_{i} \lambda_{i} \Phi\left(u_{i}\right)-\sum_{j} \mu_{j} \Phi\left(v_{j}\right)\right\|+\left\|\sum_{i} \lambda_{i} \Psi\left(u_{i}\right)-\sum_{j} \mu_{j} \Psi\left(v_{j}\right)\right\|
\end{aligned}
$$

The first member of this last sum is less than $\varepsilon / 2$ because of the $\sigma$-slice continuity. In fact, using the inequalities (5) and (7) we have

$$
\begin{aligned}
\left\|\sum_{i} \lambda_{i} \Phi\left(u_{i}\right)-\sum_{j} \mu_{j} \Phi\left(v_{j}\right)\right\| & \leqslant\left\|\sum_{i} \lambda_{i}\left(\Phi\left(u_{i}\right)-\Phi(x)\right)\right\|+\left\|\sum_{j} \mu_{j}\left(\Phi\left(v_{j}\right)-\Phi(x)\right)\right\| \\
& \leqslant\left(\sum_{i \notin I_{0}} \lambda_{i}+\sum_{j \notin J_{0}} \mu_{j}\right) \frac{1}{8 m}+\left(\sum_{i \in I_{0}} \lambda_{i}+\sum_{j \in J_{0}} \mu_{j}\right) 2 M_{k, q} \\
& <\frac{1}{4 m}+2\left(\delta_{x}+s_{x}\right) \frac{1}{r_{x}} 2 M_{k, q} \\
& <\frac{1}{4 m}+\frac{1}{2 m}=\frac{3}{4 m}<\frac{\varepsilon}{2} .
\end{aligned}
$$

To obtain the same bound for the second summand in (8) we use the midpoint locally uniformly rotund decomposition in the beginning of the proof. Let us consider the vectors in $c o\left(Y^{n, m}\right): u^{\prime}=\sum_{i} \lambda_{i} \Psi\left(u_{i}\right)$ and $v^{\prime}=\sum_{j} \mu_{j} \Psi\left(v_{j}\right)$. Now, bearing in mind that $\Psi(x)$ is an $(\varepsilon / 2,1 / m)$-strongly extreme point of $c o\left(Y^{n, m}\right)$, if we are able to prove that $\| \Psi(x)$ $-\left(u^{\prime}+v^{\prime}\right) / 2 \|<1 / m$, we can conclude that $\left\|u^{\prime}-v^{\prime}\right\|<\varepsilon / 2$ and finish the proof. 
The next inequalities give the estimation wanted:

$$
\begin{aligned}
\left\|\Psi(x)-\frac{u^{\prime}+v^{i}}{2}\right\| & =\left\|x-\frac{u+v}{2}-\frac{1}{2} \sum_{i} \lambda_{i}\left(\Phi(x)-\Phi\left(u_{i}\right)\right)-\sum_{j} \mu_{j}\left(\Phi(x)-\Phi\left(v_{j}\right)\right)\right\| \\
& \leqslant\left\|x-\frac{u+v}{2}\right\|+\frac{1}{2}\left\|\sum_{i} \lambda_{i}\left(\Phi\left(u_{i}\right)-\Phi(x)\right)+\sum_{j} \mu_{j}\left(\Phi\left(v_{j}\right)-\Phi(x)\right)\right\| \\
& <\delta_{x}+\frac{3}{4 m}<\frac{1}{m} .
\end{aligned}
$$

\section{REFERENCES}

[1] G. Alexandrov, 'On the three space problem for MLUR renorming of Banach spaces', $C$. R. Acad. Bulgare Sci. 42 (1989), 17-20.

[2] G. Alexandrov and I. Dimitrov, 'On equivalent weakly midpoint locally uniformly rotund renormings of the space $\ell_{\infty}$ ', (in Russian), in Math. and Math. Education, Proc. 14th Spring Conference of the Union of Bulg. Mathematicians, Sunny Beach (Blgar. Akad. Nauk, Sofia, 1985,), pp. 189-191.

[3] R. Deville, G. Godefroy and V. Zizler, Smoothness and renormings in Banach spaces, Pitman Monographs and Surveys in Pure and Appl. Math. 64 (Longman Scientific and Technical, Harlow, 1993).

[4] G. Godefroy, S. Troyanski, J. Whitfield and V. Zizler, 'Three space problem for Iocally uniformly rotund renormings of Banach spaces', Proc. Amer. Math. Soc. 94 (1985), 647-652.

[5] R. Haydon, 'Trees in renorming theory', Proc. London Math. Soc. 78 (1999), 541-585.

[6] Z. Hu, W.B. Moors and M.A. Smith, 'On a Banach space without a weak mid-point locally uniformly rotund norm', Bull. Austral. Math. Soc. 56 (1997), 193-196.

[7] M. Jimnez Sevilla and J.P. Moreno, 'Renorming Banach spaces with the Mazur Intersection Property', J. Funct. Anal. 144 (1997), 486-504.

[8] K. Kunen and H. Rosenthal, 'Martingale proofs of some geometrical results in Banach space theory', Pacific J. Math. 100 (1982), 153-175.

[9] A. Molt, J. Orihuela and S. Troyanski, 'Locally uniformly rotund renorming and fragmentability', Proc. London Math. Soc. 75 (1997), 619-640.

[10] A. Molt, J. Orihuela, S. Troyanski and M. Valdivia, 'Midpoint locally uniformly rotundity and a decomposition method for renorming', Q. J. Math. 52 (2001), 181-193.

[11] A. Molt, J. Orihuela, S. Troyanski and M. Valdivia, 'A non linear transfer technique', (Prepublicaciones del Departamento de Matemticas de la Universidad de Murcia no. 20, 2003).

[12] M. Raja, 'On locally uniformly rotund norms', Mathematika 46 (1999), 343-358.

Departamento de Matemticas

Universidad de Castilla La Mancha

Escuela Politcnica Superior de Albacete

Edificio Infante Don Juan Manuel

Campus Universitario

02071 Albacete

Spain

email: sebastian.lajara@uclm.es

\author{
Departamento de Matemticas \\ Universidad de Murcia \\ Campus de Espinardo \\ 30100 Murcia \\ Spain \\ e-mail: apall@um.es
}

